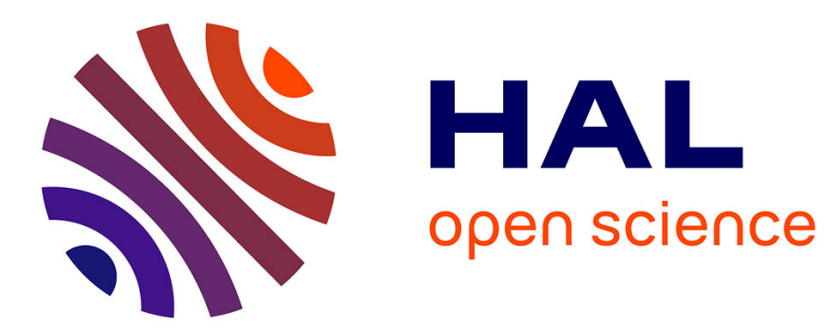

\title{
Analysis of a Turbine Bladed Disk With Structural and Aerodynamic Mistuning
}

Dimitri Franz, Loic Salles, Sina Stapelfeldt

\section{To cite this version:}

Dimitri Franz, Loic Salles, Sina Stapelfeldt. Analysis of a Turbine Bladed Disk With Structural and Aerodynamic Mistuning. ASME Turbo Expo 2017: Turbomachinery Technical Conference and Exposition, Jun 2017, Charlotte, United States. 10.1115/GT2017-64586 . hal-02544648

\section{HAL Id: hal-02544648 \\ https://hal.science/hal-02544648}

Submitted on 16 Apr 2020

HAL is a multi-disciplinary open access archive for the deposit and dissemination of scientific research documents, whether they are published or not. The documents may come from teaching and research institutions in France or abroad, or from public or private research centers.
L'archive ouverte pluridisciplinaire HAL, est destinée au dépôt et à la diffusion de documents scientifiques de niveau recherche, publiés ou non, émanant des établissements d'enseignement et de recherche français ou étrangers, des laboratoires publics ou privés. 


\section{ANALYSIS OF A TURBINE BLADED DISK WITH STRUCTURAL AND AERODYNAMIC MISTUNING}

\section{Dimitri Franz}

Royal Institute of Technology

Department of Energy Technology

14128 Stockholm

Sweden

\author{
Loic Salles, Sina Stapelfeldt
}

Vibration University Technology Centre
Mechanical Engineering Department
Imperial College London
London SW7 2AZ
United Kingdom

\section{ABSTRACT}

In this paper the effects of mistuning on the flutter stability of a turbine blade are analysed. Two types of mistuning are considered, frequency mistuning and aerodynamic mistuning. The study concentrates on the the first family of modes $(1 F$, first flap) as the blade fluttered in this mode during test. For the frequency mistuning analysis, the $1 F$ frequency is varied around the annulus but the $1 F$ mode shapes remain the same for all the blades. The mistuning analyses are performed by using a reduced order model (ROM) based on an eigenvalue analysis of the linearized modal aeroelastic system with the aerodynamic matrix calculated from the aerodynamic influence coefficients. The influence coefficients required for this algorithm are obtained from a three-dimensional, non-linear aeroelastic solver (AU3D) by shaking one blade in the datum (tuned) frequency and mode and recording aerodynamic forces on the other blades in the assembly. After the ROM is validated against the non-linear method for the tuned case, it is used for the mistuning and mis-staggering study as time-domain computations of such cases are very time consuming.

The results of this paper indicate that, frequency mistuning is always stabilizing but aerodynamic mistuning can be destabilizing under certain conditions. Moreover, it is shown that the effect of frequency mistuning is much higher than the one of aerodynamic asymmetries and that structural coupling limits the effects of mistuning.

\section{NOMENCLATURE}

IBPA Interblade phase angle

ND Nodal diameter

FMM Fundamental Mistuning Model

AIC Aerodynamic influence coefficient

$\Delta \omega$ Mistuning amplitude

$\Lambda^{0} \quad$ Tuned stiffness matrix

$\Pi$ Pressure ratio

$\zeta_{\text {aero }}$ Aerodynamic damping

$\sigma \quad$ Standard deviation

$\omega^{*} \quad$ Reduced frequency, $\omega^{*}=\omega^{0} c / V$

$\omega_{s}^{0} \quad$ Tuned blade frequency of the $\mathrm{s}^{\text {th }}$ blade

$\omega_{s} \quad$ Mistuned blade frequency of the $\mathrm{s}^{\text {th }}$ blade

$\omega$ Complex system frequency

$A^{m 0} \quad$ Tuned aerodynamic influence coefficient matrix

$A_{x}^{m 0} \quad$ Tuned aerodynamic influence coefficient matrix in physical space

$A_{x}^{m} \quad$ Aerodynamic matrix containing tuned and mistuned forces in physical space

$\hat{A}^{m} \quad$ Mistuned aerodynamic matrix

$\hat{A} \quad$ Frequency mistuned matrix

$E \quad$ Discrete Fourier transformation matrix

$E^{*} \quad$ Hermitian of the discrete Fourier transformation matrix

$I$ Identity matrix (tuned mass matrix)

$F$ Modal force

$N$ Blade number

$a_{s}^{0} \quad$ Tuned aerodynamic influence coefficient on the $\mathrm{s}^{\text {th }}$ blade

$f_{b a}^{0} \quad$ Tuned blade alone frequency

$q$ Travelling wave displacement 


\section{INTRODUCTION}

Structural or aerodynamic mistuning, i.e. deviations from the design intent, in turbomachinery bladed disks and blisks are caused by manufacturing tolerances and/or wear. It has been long known that they affect the flutter stability of bladed assemblies (see, for example, $[1,2]$ ). In fact, intentional mistuning has been used to successfully suppress flutter in both compressor and turbine rotors $[3,4]$.

The vast majority of mistuning studies have focused on structural mistuning and been neglecting any aerodynamic coupling between blades. High fidelity studies incorporating both structural and aerodynamic coupling and mistuning effects have been comparatively rare [5]. Recently, Kielb et al. [5,6] investigated the effects of frequency and aerodynamic mistuning on the flutter stability of a front-stage compressor blisk. The results indicated that, frequency mistuning and certain patterns of aerodynamic asymmetry were able to suppress flutter but that random aerodynamic asymmetries destabilized the blades.

This paper uses the same approach to study the effect of mistuning on the flutter stability of a low pressure turbine rotor. The bladed-disk rotor was designed by ITP and tested by CTA as part of the European Collaborative project FUTURE [4,7] and is representative of a state-of-the-art low pressure turbine. Two types of mistuning, frequency mistuning and aerodynamic mistuning, are chosen for this study. They are analyzed using a mistuning model similar to the one developed by Kielb et al. [5]. The model is based on an eigenvalue analysis of the linearized modal aeroelastic system. It combines a reduced order structural coupling model with an aerodynamic coupling model based on aerodynamic influence coefficients (AIC) obtained from unsteady computational fluid dynamic (CFD) simulations. Previous work by the authors [8] has shown that this method predicts similar flutter boundaries for a fan blade as a non-linear CFD analysis. Aerodynamic mistuning is modeled by introducing an aerodynamic asymmetry (such as would arise from circumferential stagger angle variations, for example) in the aerodynamic coupling model.

For simplicity, the term reduced order model (ROM) will be used to refer to the model based on Fundamental Mistuning Model with Aerodynamic Influence Coefficients.

The work is divided into three parts. First, the flutter stability of the tuned assembly in the first flap (1F) mode is investigated. Three-dimensional, non-linear URANS simulations are used to determine the influence of loading on the aeroelastic stability of the rotor blade. The results are also used to validate the ROM for the tuned system. In the second part, the ROM is used to study the effects of frequency mistuning on aero-damping. Finally, the sensitivity of the system to aerodynamic asymmetries is studied while frequency mistuning is set to zero.

\section{TEST CASE}

This study is performed on a stand-alone low pressure turbine bladed disk consisting of 146 blades. The blade was designed by ITP, Spain, as part of the FUTURE project - a Euro-
TABLE 1. TEST CASE SUMMARY

\begin{tabular}{lc}
\hline Design inlet Mach No & 0.42 \\
Design exit Mach No & 0.75 \\
Reynolds number & $1.2 \times 10^{5}$ \\
Reduced frequency, 1F & 0.075 \\
\hline
\end{tabular}

pean project aiming at improving and validating current stateof-the-art prediction tools for flutter in turbomachines. The blade is representative of a modern low pressure turbine with high deflection, high lift blading and high subsonic exit Mach number. The rotor was tested in isolation with inlet guide vanes located more than 10 chords upstream of the rotor. Important parameters of the test case are listed in Table 1. A more detailed description of the design and test can be found in References $[4,9]$.

The rotor blade was designed to be unstable in the first flap (1F) mode at the 'nominal' operating condition with exit Mach number 0.75 . The reduced frequency, based on semi-chord, exit velocity and frequency in $\mathrm{Hz}$, at this condition is 0.075 .

\section{COMPUTATIONAL APPROACH Flow solver}

The flow solver used throughout the aeroelastic analysis is AU3D which was written in Imperial College with support from Rolls-Royce. The code has been used and validated against experimental data for over two decades (see [10,11], for example). The code models the 3D unsteady Reynoldsaveraged Navier-Stockes equations. All computations in this paper are performed using the one-equation Spalart-Allmarars turbulence model [12]. The applied solution method is implicit with second-order accuracy in space and time. Details about the flow solver can be found in Reference [13].

\section{Computational domain}

The turbine rotor was modeled in isolation without inlet guide vanes. The blade was meshed using an in-house mesh generator [14]. The resulting grid is structured in the radial direction and unstructured in the blade-to-blade direction. For the purpose of this study, the hub and tip walls were modeled inviscid. This was not expected to influence the flutter results, as the blade has a large aspect ratio, end-wall effects are negligible and the flow over the turbine is effectively two-dimensional. The computational domain was extended upstream and downstream of the rotor with a gradually coarsening grid to minimize numerical reflections from the boundaries. The final grid contained $45 \mathrm{k}$ nodes per blade passage.

\section{Aeroelastic method}

To analyze the tuned and mistuned assemblies, a variety of methods is used: Fully-coupled full circumference simulations, 
an energy method using single-passage domains with phaselagged boundary conditions and a reduced order method using aerodynamic influence coefficients obtained from a partial assembly aeroelastic simulation.

In all cases, material damping is set to zero because the exact value is unknown for the current rotor configuration but its expected value is much smaller than the estimated aerodynamic damping. This paper uses the usual sign convention where a positive ND represents a forward traveling wave, i.e. a wave traveling in the positive direction of rotation, and a negative ND a backward traveling wave.

All unsteady flutter simulations were started from converged steady solutions.

Weakly-coupled full circumference simulations For this method, the computational domain includes all 146 blades. The blade mode shape is expanded to nodal diameters (NDs) and the flutter computation is started by giving the blades an initial velocity in the expanded modes. At each time step, the forces acting on the blade and the resulting blade displacement is calculated. Note that the mode shape remains fixed and is not altered by the aerodynamics. For this reason, this method is sometimes called 'weakly-coupled'. Tracking the time history of displacement allows to determine the system stability by using the logarithmic decrement method. The aerodynamic damping is obtained for all ND at once. This method will be referred to as ND expansion method. It can model rigid and flexible disks and will be used in this paper to (a) determine the importance of structural coupling and (b) determine the most unstable nodal diameter in the tuned assembly.

\section{Single passage with phase-lagged boundary con-}

ditions To reduce computational efforts, some of the conditions are modeled using a single-passage method with phaselagged boundary conditions. In this case, the blade is oscillating at a constant amplitude and the aerodynamic damping is calculated from the work done on the blade by the air. The nodal diameter, or inter-blade phase angle (IBPA), is imposed via the phase-lagged boundary conditions. This method assumes a rigid disk without structural coupling between blades.

Reduced order method with aerodynamic influence coefficients For the mistuned assemblies, an aerodynamic influence coefficient (AIC) method is used. AIC is a structural reduced order model initially developed by Feiner \& Griffin [15] and extended by Kielb et al. [5] for aerodynamic coupling. The initial model, known as fundamental mistuning model (FMM), requires two sets of input parameter in order to solve for the system stability: the natural frequency and the deviation of frequency due to mistuning. In this method the unsteady aerodynamics are uncoupled from the structural analysis. This approach is common and valid for high mass ratio blades. To account for blade coupling forces, CFD simulations are performed in conjunction with cascade aerodynamic theory. The stability is determined by solving the eigenvalue problem of the aeroelastic system in the traveling wave space.

$$
\left[\boldsymbol{\Lambda}^{0}+\hat{\boldsymbol{A}}-\boldsymbol{A}^{m 0}-\hat{\boldsymbol{A}}^{m}-\boldsymbol{\omega}^{2} \boldsymbol{I}\right]\{q\}=\{0\}
$$

$\Lambda^{0}$ represents the modal stiffness matrix and $\hat{A}$ the frequency mistuning matrix containing the frequency variation in each individual bladed disk sector [15]. $\hat{A}$ is circulant and its component in the $\mathrm{m}^{\text {th }}$ traveling number couples the $\mathrm{n}^{\text {th }}$ traveling wave with the $(\mathrm{n} \pm \mathrm{m})^{\text {th }}$ traveling wave. Its components can be calculated using

$$
\hat{A}_{n, m}=\frac{2 \omega_{n}^{0} \omega_{m}^{0}}{N} \sum_{s=0}^{N-1}\left[\Delta \omega_{s} e^{i(n-m) \frac{2 \pi s}{N}}\right]
$$

The fractional change in frequency $\Delta \omega_{s}$ or the mistuning amplitude is defined as

$$
\Delta \omega_{s}=\frac{\omega_{s}-\omega_{s}^{0}}{\omega_{s}^{0}}
$$

where $\omega_{s}^{0}$ and $\omega_{s}$ are the structural tuned and mistuned frequency of the $\mathrm{s}^{\text {th }}$ blade respectively.

$\boldsymbol{A}^{m 0}$ is the diagonal aerodynamic influence matrix of the tuned system. The coefficients in this matrix are obtained from the CFD simulation by shaking the reference blade in the desired modeshape and measuring the modal forces on the other blades [8]. The tuned aerodynamic influence coefficient of the $s^{\text {th }}$ blade attributed to a sector based mode is calculated by

$$
a_{s}^{0}=\frac{F_{s, \text { real }}+i F_{s, \text { imag }}}{q_{\text {ref,real }}+i q_{\text {ref,imag }}}
$$

where $F_{s}$ is the modal force on the blade $\mathrm{n}$ due to displacement of the reference blade and $q_{r e f}$ is the modal displacement of the reference blade. In this analysis, in order to calculate the harmonic coefficients, a fast Fourier transformation of the unsteady AU3D simulations results is taken. The transformation of the aerodynamic influence coefficients matrix of the second based mode to the traveling space is done by a discrete Fourier transformation

$$
\boldsymbol{A}^{m 0}=\frac{1}{N} \boldsymbol{E}^{*} \boldsymbol{A}_{x}^{m 0} \boldsymbol{E}
$$

$\hat{\boldsymbol{A}}^{m}$ accounts for the aerodynamic asymmetries due to mistuning in traveling wave coordinates. Aerodynamic asymmetries are introduced in the physical space and transformed to travelling wave space in the same manner as in Eq. (5) [6].

The aerodynamic damping can be calculated by taking the ratio of the real part to the imaginary part of the system frequency

$$
\zeta_{\text {aero }}=\frac{\omega_{\text {imag }}}{\omega_{\text {real }}}
$$


The real part of the complex system frequency is always positive which means that the stability is defined by the imaginary part.

\section{TUNED ANALYSIS}

The tuned frequency and modeshape of the first flap (1F) mode were determined from a finite element analysis with cyclic symmetric boundary conditions. Before the stability of the tuned system was analyzed in detail, the different numerical approaches used in this work were validated.

Validation of numerical approaches Some of the approaches used in this paper inherently assume a rigid disk without any structural coupling between blades. To validate this assumption, the level of structural coupling of the bladed disk is first assessed by determining the eigenfrequencies of a flexible blade sector for all ND. The frequency of the $1 \mathrm{~F}$ mode in a given ND is plotted as a fraction of the blade alone frequency in Fig.1.

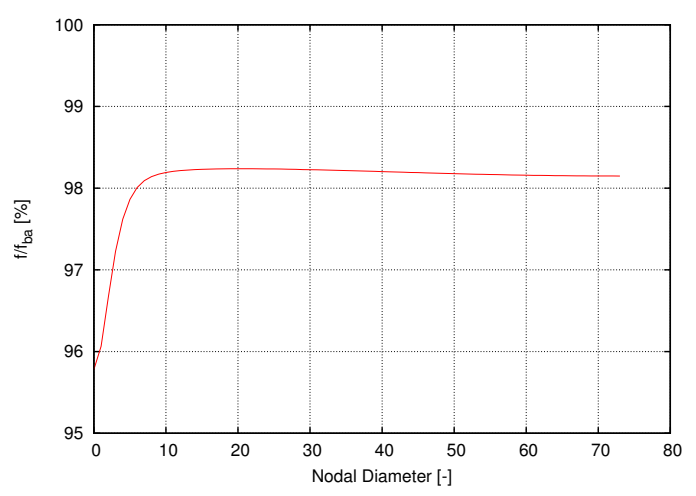

FIGURE 1. TUNED SYSTEM FREQUENCIES

It can be observed that the mode shapes are bladedominated and therefore, the strain energy is primarily in the blades for all nodal diameter. Families with blade-dominated modes are assumed to be isolated from neighbouring families. As a consequence, the modal coupling effects from other families due to frequency mistuning is negligible which is crucial for the later mistuning analysis [16].

In the next step of the validation, the aeroelastic stability was compared for the following approaches: a) ND expansion method with flexible disk, b) ND expansion method with rigid disk, c) AIC method with rigid disk and d) single passage method (rigid disk). The resulting aerodynamic damping values at the nominal operating point are shown in Fig.2.

It can be seen that all forward travelling waves are stable whereas the majority of negative waves is unstable. There is a good agreement between the different methods. All methods predict the same variation of aero-damping with ND and there are no noticeable differences between the two full annulus methods and AIC methods in terms of aerodamping. The

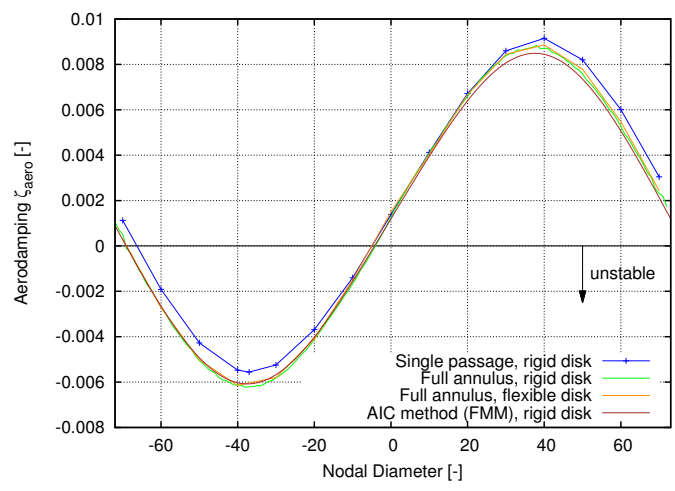

FIGURE 2. COMPARISON OF STABILITY CURVES FOR DIFFERENT METHODS

single passage, however, overpredicts the maximum and minimum aerodynamic damping values by approximately $10 \%$. The least stable ND is -37 . Although the natural frequency is decreased by about $2 \%$ for most ND when the tuned disk is considered flexible, it has almost no effect on the stability.

Effect of Loading on Stability To gain some insights into the physical mechanisms influencing the stability of the tuned system the blade loading is varied. To do so, the system stability at the least stable nodal diameter (ND -37) is examined using the single passage domain with phase-lagged boundary conditions.

For a given frequency, the stability will vary with blade loading. This effect is investigated by altering the static outlet pressure in the computations while holding the inlet stagnation pressure constant. The computed aerodynamic damping is plotted against blade loading in Fig. 3, where blade loading is expressed as a ratio of the exit pressure, $p_{2}$ to the nominal exit pressure $p_{2, r e f}$. The figure clearly shows a linear behavior for small variations in loading. In the range $0.85 \leq p_{2} / p_{2, \text { ref }} \leq 1.05$ aero-damping decreases linearly with increasing blade loading. When the outlet pressure is decreased below $80 \%$ of the nominal value, however, non-linearities arise.

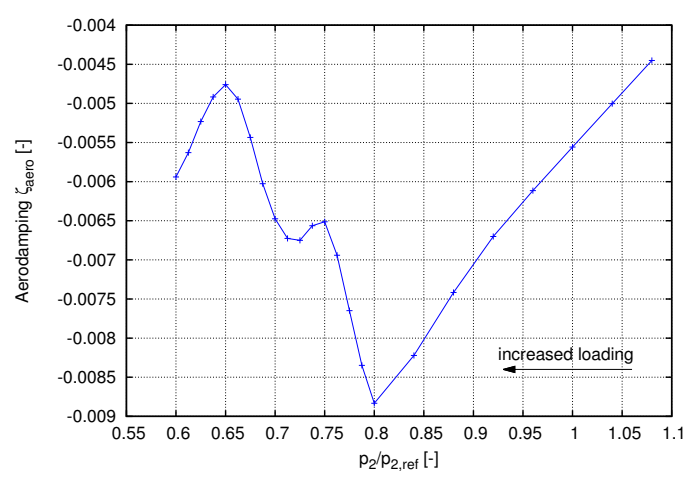

FIGURE 3. EFFECT OF BLADE LOADING ON AERODYNAMIC DAMPING 
To explore this phenomena, the flow field at three different pressure ratios, $p_{2} / p_{2, r e f}=1.0,0.8$ and 0.65 , is analysed. Fig. 4 shows the Mach number contours at the different loading conditions at $90 \%$ span. A comparison with Fig. 3, suggests that the non-linearity is due to a shock which appears at about $80 \%$ of the chord at $p_{2} / p_{r e f}=0.80$, and travels towards the trailing edge when the loading is increased further. When the shock is created, the aerodynamic damping starts to increase but drops again after the shock reaches the trailing edge $\left(p_{2} / p_{2, \text { ref }}=0.65\right)$ and a fish tail shock appears. When the back pressure is decreased below a ratio of 0.65 (not shown), the fish tail shock intensity grows causing the aerodynamic damping to decrease again as mentioned earlier.

It should be noted here that since the endwalls were modelled inviscid, the effect of secondary flows could not be captured in the computations. However, their impact on the solution might be considerable, especially for $1 \mathrm{~F}$ mode flutter, which should be studied in the future.

The aeroelastic stability of a blade is determined by the phase-lag between the blade surface velocity and the unsteady pressure on the blade surface. Looking at the phase of the unsteady pressure can thus provide more information about the cause of the aerodynamic damping change with blade loading. The phase of the unsteady pressure (with respect to that of the blade motion) is plotted along the suction side in Fig. 5 for different loading conditions. It can be seen that, the phase changes considerably with blade loading over the second half of the chord. The slope of the phase-versus-chord line is proportional of the pressure disturbance's propagational velocity along the chord and its minimum coincides with the location of the shock $\left(\approx 0.8 \mathrm{x} / \mathrm{c}\right.$ for $p_{2} / p_{2, r e f}=0.8$ and $\approx 0.8 \mathrm{x} / \mathrm{c}$ for $\left.p_{2} / p_{2, \text { ref }}=0.65\right)$. This confirms that the change in aero-damping is closely related to the appearance and movement of the shock. Note that the steady and unsteady pressures along the pressure side do not change much with loading and hence the the pressure side phase plot has been omitted for brevity.

Finally, full annulus calculations (ND expansion method) are performed in order to calculate the aerodynamic damping as a function of nodal diameter for an increased loading, Fig. 6.

For the range $1.10 \leq p_{2} / p_{\text {ref }} \leq 0.85$ the shape of the stability curve (S-curve) remains unchanged and merely the amplitude of the damping changes. In this linear range (see Fig. 3), the $\mathrm{S}$-curve is scaled with a good approximation by

$$
\zeta_{\text {aero }, \Pi} \approx \frac{1}{\Pi^{2}} \zeta_{\text {aero }, \Pi=1}
$$

where $\Pi$ is the pressure ratio. When shocks start to appear at $p_{2} / p_{\text {ref }}=0.8$, the shape of the $\mathrm{S}$-curve changes. The least and most stable nodal diameter shift to a smaller negative and positive inter-blade phase angle respectively. When the fish tail shock is established at $p_{2} / p_{\text {ref }}=0.65$, two local peaks appear in the stable range of the $\mathrm{S}$-curve.

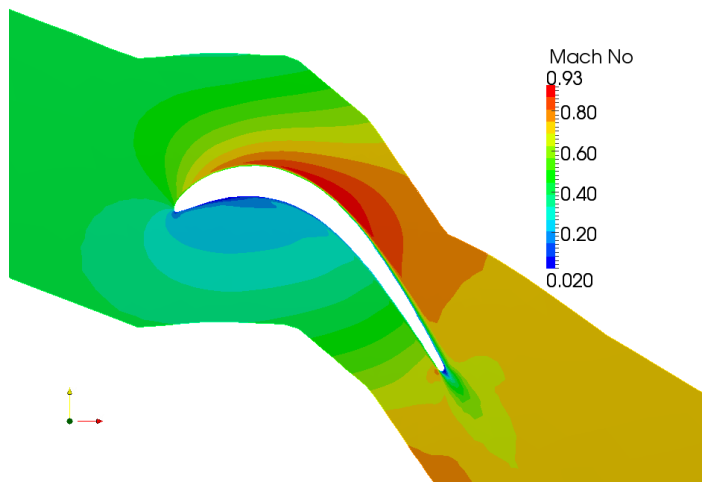

(a) $p_{2} / p_{2, r e f}=1$

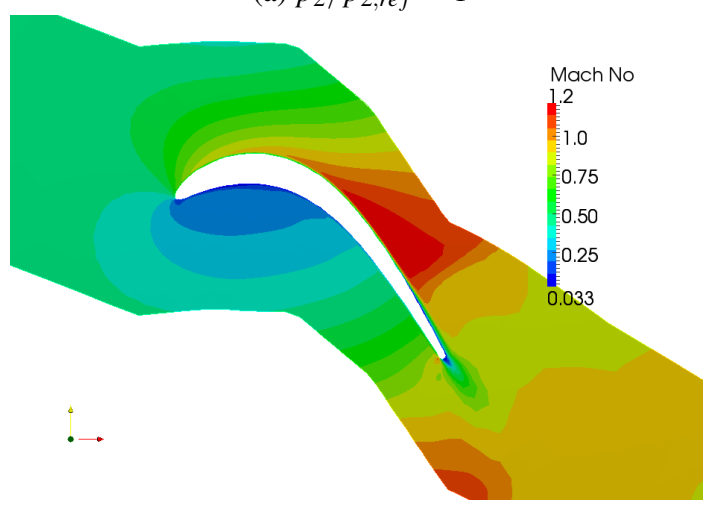

(b) $p_{2} / p_{2, r e f}=0.8$

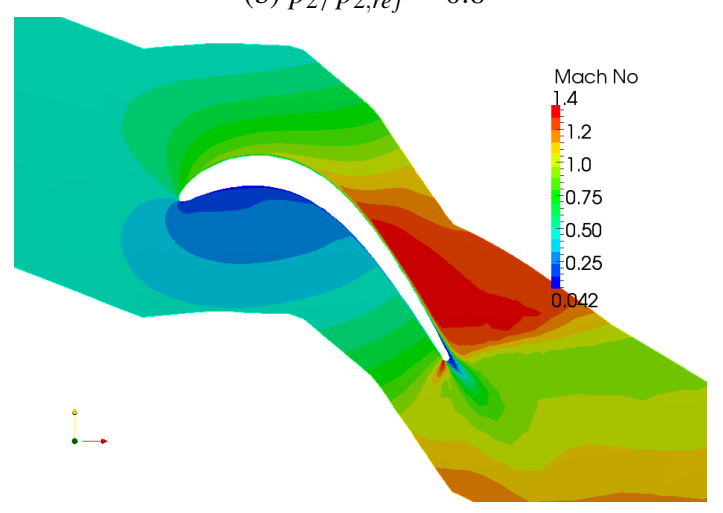

(c) $p_{2} / p_{2, \text { ref }}=0.65$

FIGURE 4. RELATIVE MACH NUMBER IN BLADE PASSAGE FOR DIFFERENT BLADE LOADINGS AT 90\% SPAN

\section{MISTUNED ANALYSIS}

The influence coefficient due to unsteady aerodynamic forces are calculated using the blade alone mode shape and frequency (without structural coupling). The same coefficients are used for all traveling waves indices and thus, the results are only valid for cases where the modeshape and frequency do not change significantly with nodal diameter.

The eigenvalue problem of the FMM can either be solved in the physical or in the traveling wave space under the assumption of a rigid disk. However, the representation of mistuning in the traveling wave space relates the mistuning to the blade and the corresponding sector of the disk. Therefore, if the mistuning is confined to the blades only, the fractional frequency 


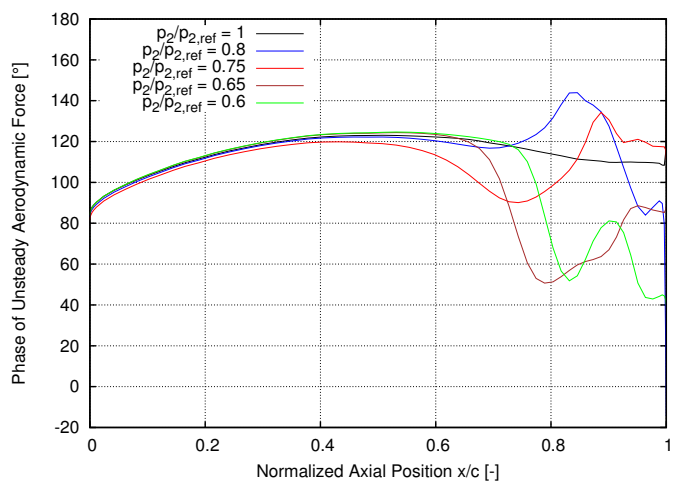

FIGURE 5. PHASE OF THE UNSTEADY PRESSURE ON THE SUCTION SIDE AT $90 \%$ SPAN

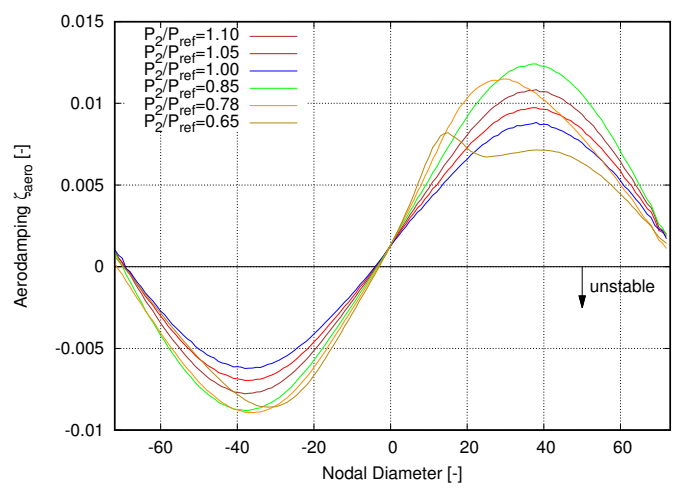

FIGURE 6. STABILITY CURVE COMPARISON FOR DIFFERENT SYSTEMS

deviation has to be scaled $[17,18]$. This error is determined by solving the eigenvalue problem while setting the aerodynamic forces to zero. In the present case the error in the prediction of the mistuned blade frequency compared to the input was found to be up to $0.3 \%$ for an alternate mistuning pattern of $7 \%$. Since the error is relatively small, the scaling is omitted in this investigation. In other words, the frequency of a sector is approximated by the blade frequency. Nevertheless, when the disk is flexible, the eigenvalue problem has to be solved in traveling wave space because the tuned natural frequencies are usually determined by a finite element analysis with cyclic symmetric boundary conditions.

For the aerodynamic asymmetry (mis-staggering) analysis the aerodynamic influence coefficients are computed for the tuned case and analytically perturbed in the eigenvalue problem. This approach permits to take into account any kind of aerodynamics asymmetries. This approach is quite similar to the non-parametric approach proposed by Mbaye et al. [19]. It does not unfortunately allow to link the amount of aerodynamic mistuning with angle mis-staggering unlike a deterministic approach [20] It gives a good understanding of aerodynamic asymmetries and their influence on rotor stability. A similar study was performed on a compressor by Kielb et al. (2007) [6] and on a fan by Miyakozawa (2007) [16].

All mistuning analysis are conducted using the linear ex- tended ROM model. The Aerodynamic Influence Coefficient matrix is calculated for the tuned system. This method was validated and successfully employed for fan flutter analysis by Salles and Vahdati (2016) [8].

\section{Frequency Mistuning}

Two different types of frequency mistuning are studied: Alternate and random mistuning.

Figure 7 shows the variation in aero-damping with mistuning amplitudes for an alternate mistuning pattern. The different lines correspond to different numbers of coefficients (AICs) used in the ROM model. In addition, each AIC was obtained with and without structural coupling between the blades. It is clear that, structural coupling (flexible disk) does not significantly affect the stability of the system. Moreover, the difference between 15 and 7 aerodynamic influence coefficient is negligible while the absolute aerodynamic damping predicted using 3 coefficients is always higher. Thus, in all further analysis 7 coefficients are considered to be sufficient. When only one coefficient is used, the system is stable because the blade will not flutter without the influence of the neighboring blades. Figure 7 shows that an alternate mistuning of $3.7 \%$ can stabilize the system.

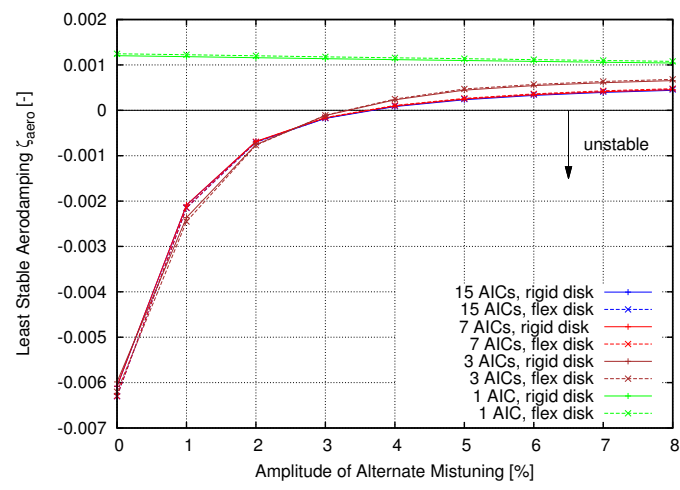

FIGURE 7. LEAST DAMPING SENSITIVITY TO NUMBER OF AIC FOR ALTERNATE MISTUNING

The aerodynamic damping versus the aeroelastic frequency of the system is plotted in Fig. 8 for a rigid disk (no structural coupling) and a flexible disk (with structural coupling). The system frequency is normalised by the tuned blade alone frequency.

For both cases, alternate mistuning introduces a frequency split in the root loci distribution. The frequency split is caused by the lack of pure traveling wave modes since they are coupled among each other. For an alternate mistuning in particular, the $n^{\text {th }}$ traveling wave mode is coupled with the $\left(n+\frac{N}{2}\right)$ mode for an even blade count. So the most unstable ND -37 of the tuned case is now coupled with a stable ND 35 for the mistuned rotor. As the mistuning amplitude increases, all the aerodynamic damping tend to converge to a value which corresponds to the average of the tuned damping (see also [21]). 


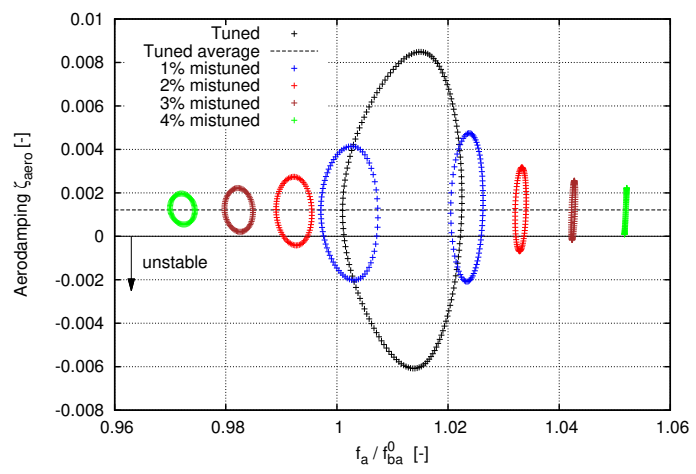

(a) WITHOUT STRUCTURAL COUPLING

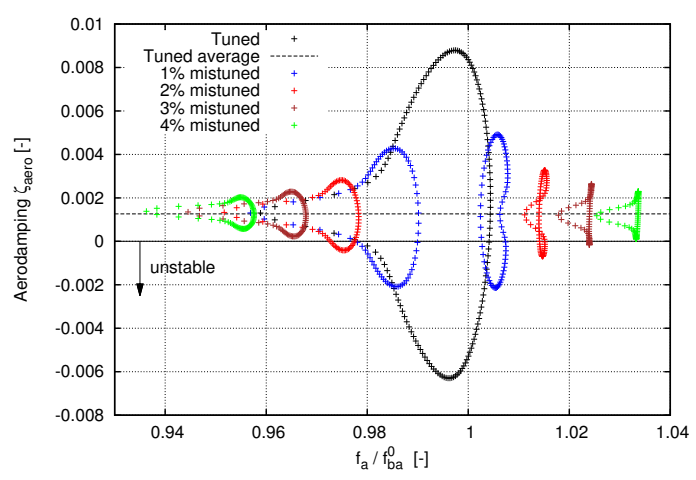

(b) WITH STRUCTURAL COUPLING

FIGURE 8. ROOT LOCI DISTRIBUTION FOR ALTERNATE MISTUNING

Both parts of the split root loci are "round" for a rigid disk, whereas some of the aeroelastic eigenvalues are shifted to the left for a flexible disk.

In general, the aeroelastic frequency corresponding to a specific coupled wave (nodal diameter) is always lower when flexibility of the disk is taken into account. The lower aeroelastic frequencies are attributed to the lower nodal diameter. The eigenfrequency increases w.r.t the nodal diameter as illustrated in Fig. 1.

To study the influence of random frequency mistuning, a Monte Carlo analysis with 1000 mistuned configurations is conducted. The frequency perturbation is assumed to be normally distributed. The population mean is the blade alone frequency for the rigid disk and the individual cyclic symmetric frequencies from the structural analysis (see Fig. 1) for the flexible disk. The least stable aero-damping for different levels of standard deviation are compared against each other and the tuned case in Fig. 9.

It is clear that the rotor will never become stable if frequency deviations are less than $\sigma=1 \%$, regardless of whether structural coupling is considered or not. This is the standard deviation which can be expected from manufacturing tolerances [22]. Fig. 9 furthermore shows that the least stable aerodynamic damping of a randomly mistuned assembly is always higher than that of a tuned assembly. The fact that more configurations of the rigid disk than of the flexible disk are stable for

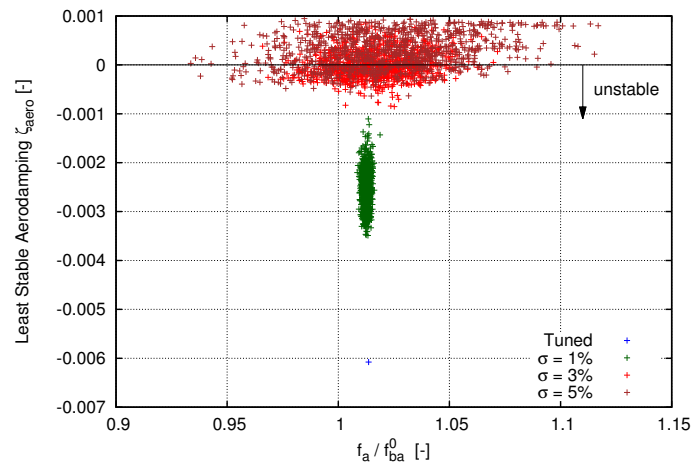

(a) WITHOUT STRUCTURAL COUPLING

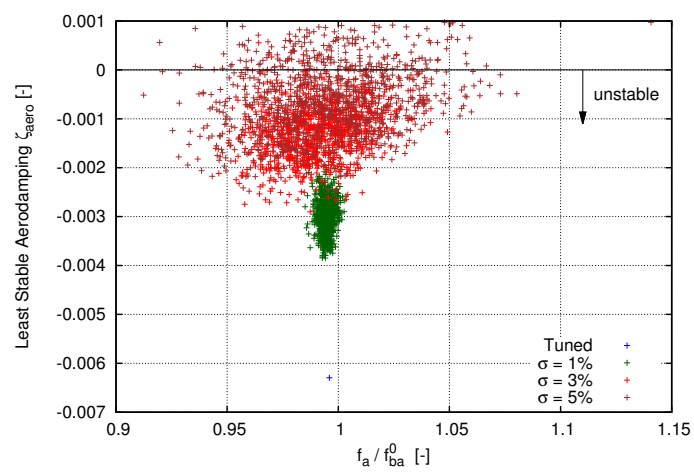

(b) WITH STRUCTURAL COUPLING

FIGURE 9. LEAST STABLE DAMPING FOR RANDOM FREQUENCY MISTUNING

the same mistuning amount indicates that structural coupling is important for frequency mistuning.

The cumulative probability of the Monte Carlo analysis, shown in Fig. 10, also demonstrates the significance of structural coupling.

A standard deviation of $3 \%$, for instance, causes about $50 \%$ of the cases to be stable when structural coupling is neglected. When structural coupling is included, however, almost none of the cases are stable. In the present case, the flexiblity of the disk is not very pronounced (see Fig. 1) but it nevertheless makes a considerable difference for randomly perturbed frequencies. This highlights the importance of structural coupling in the stability analysis of mistuned assemblies.

When the standard deviation is increased to an artificial amount, a plateau or saturation exists where a higher standard deviation does not lead to a higher aerodynamic damping. This is illustrated in Fig. 11 where the least stable aero-damping value for standard deviations up to $\sigma=50 \%$ is shown. After a certain standard deviation, most of the configurations fall on a line with a lower damping for a higher frequency. Here, the blades are relatively stiff so that the aerodynamic effects become smaller.

\section{Aerodynamic Asymmetries}

To study the effect of aerodynamic asymmetries on aerodamping the frequency mistuning is set to zero. Structural 


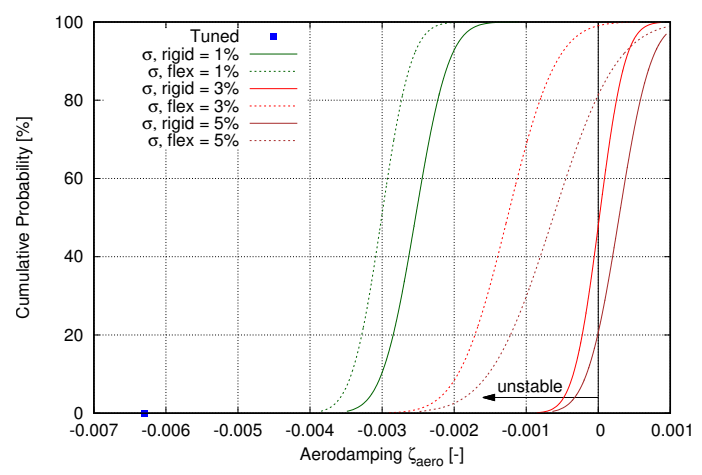

FIGURE 10. CUMULATIVE PROBABILITY OF LEAST DAMPED MODE FOR FREQUENCY MISTUNING

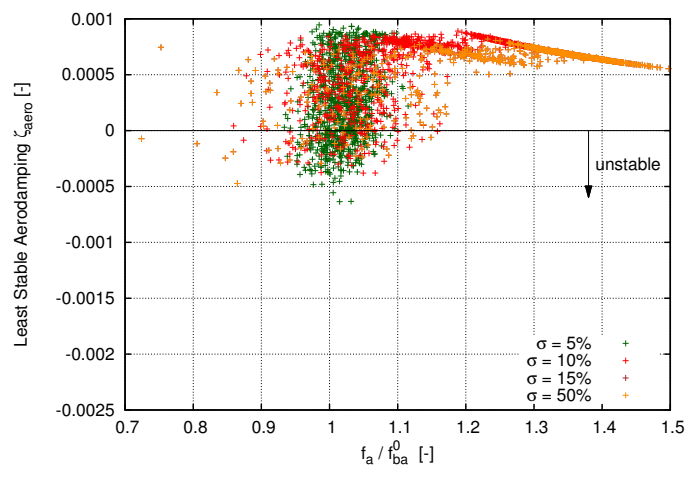

(a) WITHOUT STRUCTURAL COUPLING

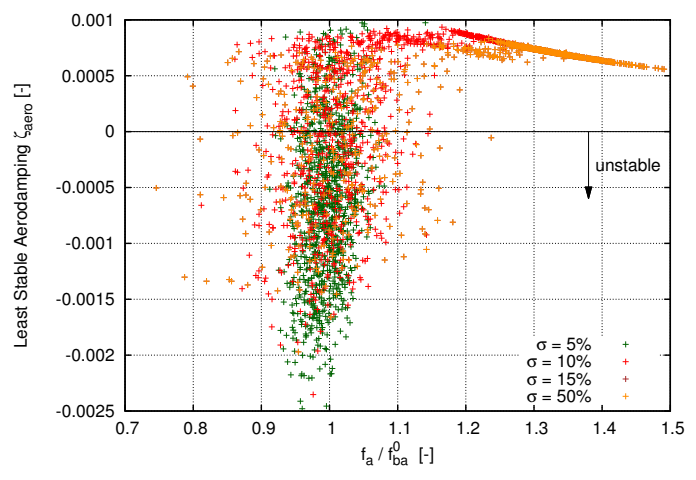

(b) WITH STRUCTURAL COUPLING

FIGURE 11. LEAST STABLE DAMPING FOR RANDOM FREQUENCY MISTUNING WITH HIGH STANDARD DEVIATION

coupling is included throughout all aerodynamic perturbation analyses. Three different types of aerodynamic mistuning are considered while the levels of mistuning are deliberately set to high levels in order to better study its effects. First, a single blade, namely blade 0 or the reference blade, is perturbed. Blade 0 is represented in the first column and first line of the AIC matrix, Eq. (8). For this type of aerodynamic asymmetry two cases are considered. One extreme case, where blade 0 is considered to be aerodynamically invisible. In order to remove blade 0 aerodynamically, all coefficients in the first column and first line of the AIC matrix are set to zero. In the second case, the coefficients are scaled by a certain amount.

$$
\boldsymbol{A}_{x}^{m}=\boldsymbol{A}_{x}^{m 0}+\hat{\boldsymbol{A}}_{x}^{m}=\left[\begin{array}{ccccc}
a_{e, e}^{0} & a_{e, o}^{1} & a_{e, e}^{2} & \ldots & a_{e, o}^{N-1} \\
a_{o, e}^{N-1} & a_{o, o}^{0} & a_{o, e}^{1} & \ldots & a_{o, o}^{N-2} \\
a_{e, e}^{N-2} & a_{e, o}^{N-1} & a_{e, e}^{0} & \ldots & a_{e, o}^{N-3} \\
\vdots & \vdots & \vdots & \ddots & \vdots \\
a_{o, e}^{1} & a_{o, o}^{2} & a_{o, e}^{3} & \ldots & a_{o, o}^{0}
\end{array}\right]
$$

The second type of aerodynamic mistuning creates an alternate pattern. The coefficient for even and odd blades are perturbed in the AIC matrix in the physical space. The coefficients with subscripts $(e, e)$ and $(o, o)$ are scaled in the complex plane resulting in a block circulant matrix. Similar to alternate frequency mistuning, the aerodynamic symmetry group perturbation has an alternate pattern which couples the $n^{\text {th }}$ mode with the mode $\left(n+\frac{N}{2}\right)$. The entries of AIC matrix in traveling space are therefore

$$
\left[A^{m}\right]=\left[\begin{array}{cc}
a^{n, n} & a^{n, n+\frac{N}{2}} \\
a^{n+\frac{N}{2}, n} & a^{n+\frac{N}{2}, n+\frac{N}{2}}
\end{array}\right]
$$

The third perturbation type is random scaling of the coefficients in the complex plane of the physical space. The real and the imaginary part are perturbed based on a normal distribution while they are assumed to be uncorrelated. As for random frequency mistuning, a Monte Carlo analysis with 1000 configurations is conducted.

The result for the first perturbation type is plotted in Fig. 12

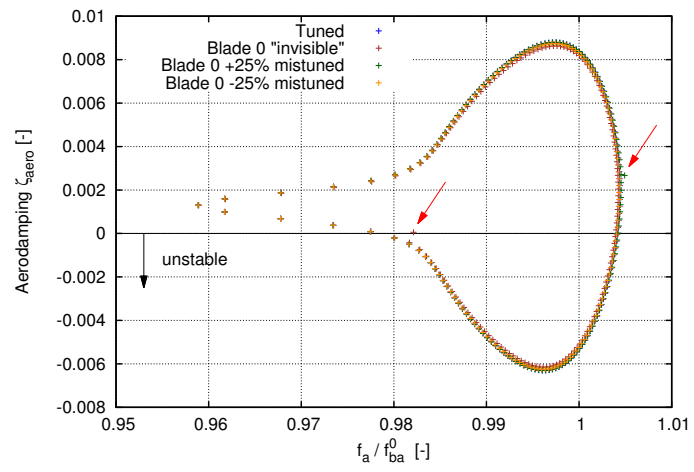

FIGURE 12. ROOT LOCI DISTRIBUTION, BLADE 0 MISTUNED

Making the blade 0 aerodynamically invisible gives a $2.3 \%$ higher damping. Reducing the force of blade 0 by $25 \%$ results in a $0.8 \%$ higher damping and increasing the force by $25 \%$ in 
$0.1 \%$ lower damping. There are two frequencies marked with a red arrow in Fig. 12 that lie outside the root loci distribution. The first one is for an invisible blade 0 and the second one for $a+25 \%$ perturbation of blade 0 . The marked frequency for an aerodynamically invisible blade 0 (left arrow in Fig. 12) is a localized blade 0 mode as can be concluded from its eigenvector, Fig. 13a.

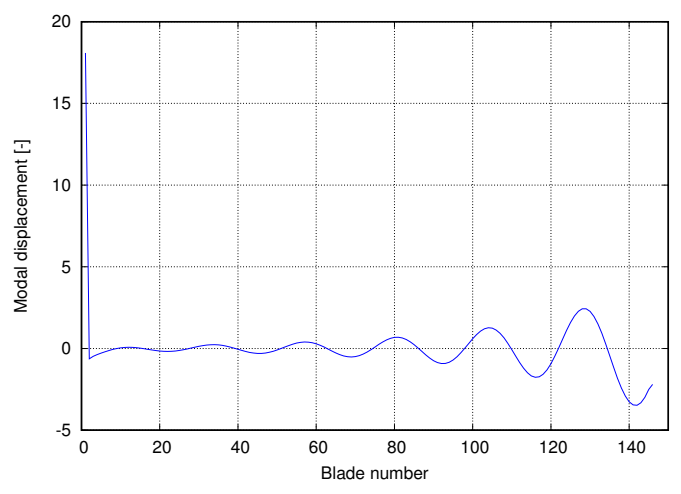

(a) LOCALIZED MODE, BLADE 0 "INVISIBLE"

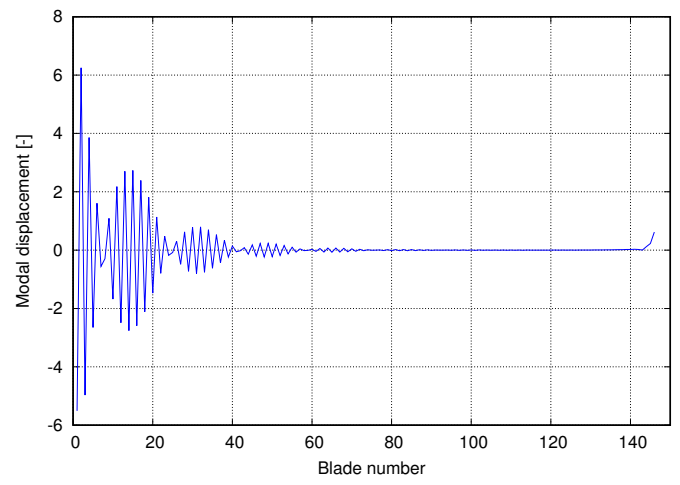

(b) MODE BLADE $0+25 \%$ MISTUNED

FIGURE 13. EIGENVECTORS FOR THE CASES OF MISTUNED BLADE 0

The marked frequency of the perturbed blade 0 (see red arrow on the right of Fig. 12) gives an eigenvector in which all blades in positive circumferential direction are displaced whereas almost all blades in negative direction are at rest, Fig. 13b. In other words, pressure perturbation of this mode is caused by blade 0 and travels in positive direction of rotation, gets weaker until it dies out. The blades in negative circumferential direction from blade 0 experience a relative weak force.

An alternate aerodynamic asymmetry increases the least stable damping by $2.1 \%$ and $8.6 \%$ for an mistuning amplitude of $10 \%$ and $20 \%$ respectively, Fig. 14.

The Monte Carlo analysis of the random stagger mistuning and the corresponding cumulative probability curve are shown in Fig. 15.

It can be observed that a random aerodynamic mistuning with a low standard deviation (here up to $10 \%$ ) can reduce the least stable damping compared to a tuned case. This means

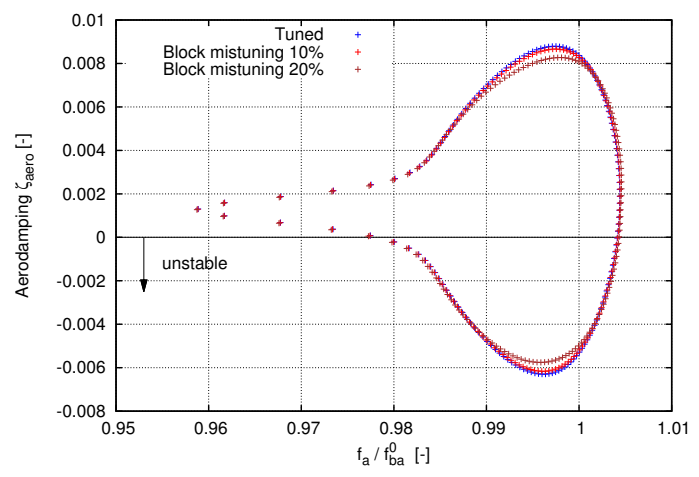

FIGURE 14. ROOT LOCI DISTRIBUTION, SYMMETRY GROUP PERTURBATION

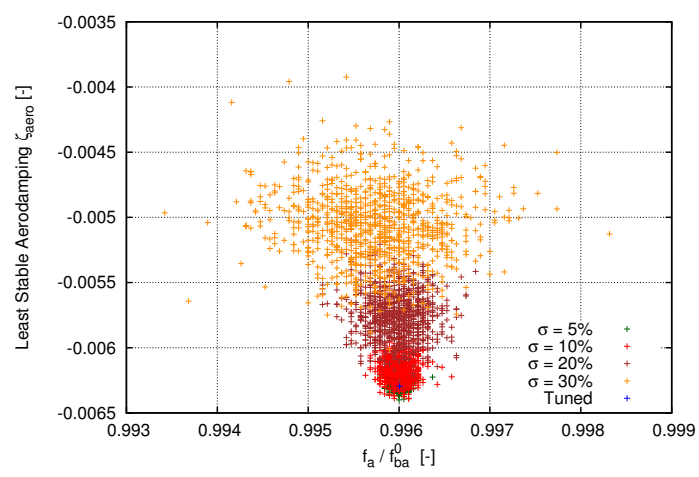

(a) MONTE CARLO RESULTS

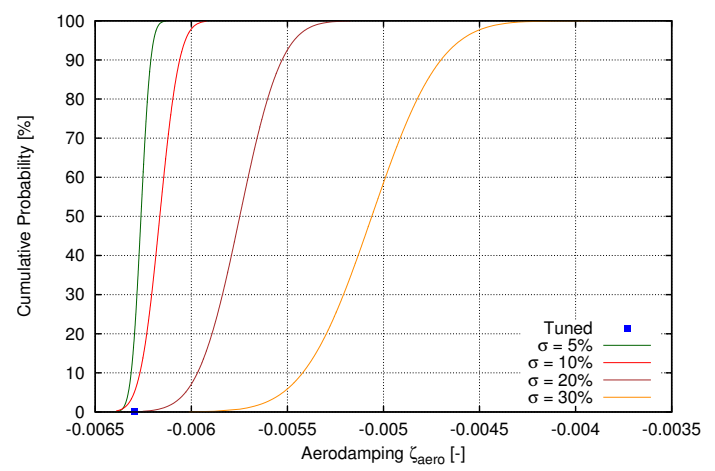

(b) CUMULATIVE PROBABILITY

\section{FIGURE 15. RANDOM AERODYNAMIC ASYMMETRIES}

that a stable system can become unstable when aerodynamic asymmetries are taken into account. However, when the structure and the aerodynamic forces of the system are randomly perturbed, the system will most likely experience a stabilizing effects because the stabilization due to frequency mistuning is higher than the destabilization caused by aerodynamics asymmetries.

\section{CONCLUSIONS AND FUTURE WORK}

This paper studied the influences of structural (frequency) and aerodynamic mistuning on the aeroelastic stability of a tur- 
bine bladed disk using a reduced order model with and without structural coupling. It was found that, frequency mistuning was always stabilizing while aerodynamic mistuning was destabilizing under certain conditions. Moreover, the results demonstrated that structural coupling can reduced the stabilizing effects of frequency mistuning. This is in agreement with findings by Kielb et al. [5] for a compressor blisk.

Future work based on this paper may include the study of perturbation of the modeshape and mis-staggering of the blade in the computational domain rather inducing frequency mistuning and aerodynamic asymmetries in the reduced order model respectively. This approach would be more realistic but also more computationally challenging. In addition, the effect of shocks, e.g. due to increased blade loading, should be study in more depth.

\section{REFERENCES}

[1] Bendiksen, O., 1984. "Flutter of mistuned turbomachinery rotors". Journal of Engineering for Gas Turbines and Power, 106(1), pp. 25-33.

[2] V. Kaza, K. R., and Kielb, R. E., 1982. "Flutter and response of a mistuned cascade in incompressible flow". AIAA Journal, 20(8), pp. 1120-1127.

[3] Schoenenborn, H., Junge, M., and Retze, U., 2012. “Contribution of free and forced vibration analysis of an intentionally mistuned blisk". In ASME Turbo Expo 2012: Turbomachinery Technical Conference and Exposition, ASME, pp. GT2012-68683.

[4] Corral, R., Beloki, J., Calza, P., and Elliot, R., 2016. "Flutter generation and control using mistuning in a turbine rotating rig". In ASME Turbo Expo 2016: Turbomachinery Technical Conference and Exposition, American Society of Mechanical Engineers, pp. V07BT34A026V07BT34A026.

[5] Kielb, R. E., Feiner, D. M., Griffin, J. H., and Miyakozawa, T., 2004. "Flutter of Mistuned Bladed Disks and Blisks with Aerodynamic and FMM Structural Coupling". Proceedings of the ASME Turbo Expo 2004, 6, pp. 573-579.

[6] Kielb, R. E., Feiner, D. M., Griffin, J. H., and Miyakozawa, T., 2007. "The Effect of Unsteady Aerodynamic Asymmetric Perturbations on Flutter". Proceedings of the ASME Turbo Expo 2007, 5, pp. 649-654.

[7] Future project website. http://www.future-project.eu/. Accessed: 2016-11-20.

[8] Salles, L., and Vahdati, M., 2016. "Comparison of Two Numerial Algorithms for Computing the Effects of Mistuning of Fan Flutter". Proceedings of the ASME Turbo Expo 2016.

[9] Corral, R., 2015. "Flutter Generation and Control in Captivity". In Proceedings of ISUAAAT14: Aeroacoustics \& Aeroelasticity of Turbomachines.

[10] Breard, C., Green, J., Vahdati, M., and Imregun, M., 2001. "A non-linear integrated aeroelasticity method for the prediction of turbine forced response with friction dampers". International Journal of Mechanical Sciences, 43, pp. 2715-2736.

[11] Sayma, A., Vahdati, M., Green, J., and Imregun, M., 1998. "Whole-assembly flutter analysis of a low pressure turbine blade". In Unsteady Aerodynamics and Aeroelasticity of Turbomachines, SPRINGER, pp. 347-359.

[12] Spallart, P., and Allmaras, S., 1992. "A One-Equation Turbulence Model for Aerodynamic Flows". AIAA Paper No. 92-0439.

[13] Sayma, A. I., Vahdati, M., Sbardella, L., and Imregun, M., 2000. "Modeling of Three-Dimensional Viscous Compressible Turbomachinery Flows Using Unstructured Hybrid Grids". AIAA Journal, 38(6), pp. 945954.

[14] Sbardella, L., Sayma, A., and Imregun, M., 1998. "Semiunstructured mesh generator for flow calculations in axial turbomachinery blading". In Unsteady Aerodynamics and Aeroelasticity of Turbomachines. Springer, pp. 541-554.

[15] Feiner, D. M., and Griffin, J. H., 2003. "Mistuning Identification of Bladed Disks Using a Fundemental Model - Part I: Theory". ASME Journal of Turbomachinery, 126(1), pp. 150-158.

[16] Miyakozawa, T., 2008. "Flutter and Forced Response of Turbomachinery with Frequency Mistuning and Aerodynamic Assemtry". PhD thesis, Duke University.

[17] Feiner, D. M., and Griffin, J. H., 2003. "Mistuning Identification of Bladed Disks Using a Fundemental Model Part II: Application". ASME Journal of Turbomachinery, 126(1), pp. 159-165.

[18] Kielb, R. E., Feiner, D. M., Griffin, J. H., and Miyakozawa, T., 2006. "Probabilistic Flutter Analysis of a Mistuned Bladed Disks". Proceedings of the ASME Turbo Expo 2006, 5, pp. 1145-1150.

[19] Mbaye, M., Soize, C., Ousty, J.-P., and Capiez-Lernout, E., 2013. "Robust analysis of design in vibration of turbomachines". Journal of Turbomachinery, 135(2), p. 021008.

[20] Sladojevic, I., Sayma, A., and Imregun, M., 2007. "Influence of stagger angle variation on aerodynamic damping and frequency shifts". In ASME Turbo Expo 2007: Power for Land, Sea, and Air, American Society of Mechanical Engineers, pp. 683-700.

[21] Martel, C., Corral, R., and Llorens, J., 2007. "Stability Increase of Aerodynamically Unstable Rotors Using Intentional Mistuning". ASME Journal of Turbomachinery, 130(1).

[22] Capiez-Lernout, E., Soize, C., Lombard, J.-P., Dupont, C., and Seinturier, E., 2004. "Blade manufacturing tolerances definition for a mistuned industrial bladed disk". In ASME Turbo Expo 2004: Power for Land, Sea, and Air, American Society of Mechanical Engineers, pp. 307-316. 